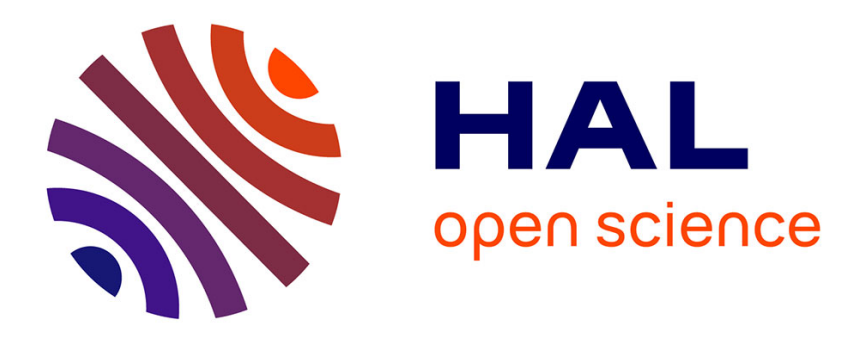

\title{
Calorimetric consequences of thermal softening in Johnson-Cook's model
}

Nicolas Ranc, André Chrysochoos

\section{To cite this version:}

Nicolas Ranc, André Chrysochoos. Calorimetric consequences of thermal softening in Johnson-Cook's model. Mechanics of Materials, 2013, 65, pp.44-55. 10.1016/j.mechmat.2013.05.007 . hal-00994498

\section{HAL Id: hal-00994498 \\ https://hal.science/hal-00994498}

Submitted on 21 May 2014

HAL is a multi-disciplinary open access archive for the deposit and dissemination of scientific research documents, whether they are published or not. The documents may come from teaching and research institutions in France or abroad, or from public or private research centers.
L'archive ouverte pluridisciplinaire HAL, est destinée au dépôt et à la diffusion de documents scientifiques de niveau recherche, publiés ou non, émanant des établissements d'enseignement et de recherche français ou étrangers, des laboratoires publics ou privés. 


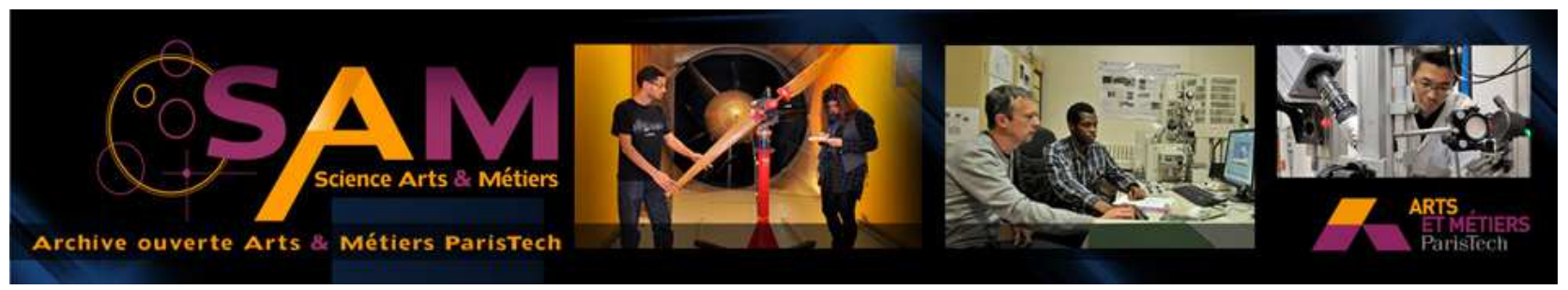

Science Arts \& Métiers (SAM)

is an open access repository that collects the work of Arts et Métiers ParisTech researchers and makes it freely available over the web where possible.

This is an author-deposited version published in: http://sam.ensam.eu Handle ID: .http://hdl.handle.net/10985/8174

\section{To cite this version :}

Nicolas RANC, André CHRYSOCHOOS - Calorimetric consequences of thermal softening in Johnson-Cook's model - Mechanics of Materials - Vol. 65, p.44-55. - 2013 


\title{
Calorimetric consequences of thermal softening in Johnson-Cook's model
}

\author{
N. Ranc ${ }^{\mathrm{a}, *}$, A. Chrysochoos ${ }^{\mathrm{b}}$ \\ a Arts et Métiers ParisTech, PIMM, UMR CNRS 8006, 151 Boulevard de l'Hôpital, F-75013 Paris, France \\ ${ }^{\mathrm{b}}$ Laboratoire de Mécanique et de Génie Civil, UMR CNRS 5508, Université Montpellier 2, CC 048, Place E. Bataillon, F-34095 Montpellier, France
}

\begin{abstract}
A B S T R A C T
At high loading rates, the development of adiabatic shear bands in metals is conventionally attributed to the strong interactions induced by viscoplastic dissipation within the bands and thermal softening effects. The rheological equation proposed by Johnson and Cook takes both viscoplastic hardening and thermal softening into account. The present paper reviews and includes this equation into a thermodynamic framework in order to analyse the energy impacts of thermal softening. Indeed this latter implies the existence of a thermomechanical coupling source, probably non-negligible and which must be considered when estimating temperature variations induced by shear band development.
\end{abstract}

\section{Introduction}

When metallic structures are loaded at high strain rate until failure, the ruin is often initiated in narrow zones where highly localized strains develop (Bai and Dodd, 1992). These thin bands of dozens of micrometers in width are called adiabatic shear bands (ASBs). Indeed, it is recognized that the transformation in these bands is close to shear mode, and the self-heating is so intense and rapid that heat has no time to diffuse, hence the term adiabatic. The link between stress and strain is often determined using the rheological equation proposed by Johnson and Cook (1983) and Johnson and Cook (1985) that accounts for both viscoplastic hardening and thermal softening induced by heat transformation of a part of the viscoplastic work.

The highly localized pattern of shear bands were attributed to the strong interactions between mechanical dissipation and the thermal softening effects (Zener and Hollomon, 1944).

\footnotetext{
* Corresponding author.

E-mail addresses: nicolas.ranc@ensam.eu (N. Ranc), andre.chrysochoos@univ-montp2.fr (A. Chrysochoos).

URL: http://pimm.paris.ensam.fr/en/user/9 (N. Ranc).
}

This autocatalytic effect can be summarized as follows: the higher the deformation rate, the more intensive the material dissipation, which consequently increases the temperature and facilitates the thermal softening and then the viscoplastic flow. The fraction of the dissipated viscoplastic work rate then plays an important role in the development of ASBs. Estimating this fraction has been and still is the focus of many studies. A review book (Bever et al.) mentions, in particular, the famous experiments performed by Taylor and Quinney (1934) which resulted in an estimate of the dissipated energy ratio of about 0.9 . This result has often been mentioned in the literature and numerous articles use the so-called Taylor-Quinney coefficient noted here $\beta_{\text {TQ }}$ (Bai and Dodd, 1992). Many other experimental studies have been indeed conducted in recent decades either at a low (Chrysochoos, 1985; Oliferuk et al., 1985) or high (Rittel, 1999) strain rate. These studies showed that $\beta_{\mathrm{TQ}}$ is generally not a "material" constant and may depends on the strain amplitude and its history and/ or on the strain rate (Rittel et al., 2009). From a theoretical standpoint, it should first be noted that the evolution of $\beta_{\mathrm{TQ}}$ can be completely determined from behavior models which are constructed and identified in a thermodynamic framework (Rosakis et al., 2000; Longère and Dragon, 2009). The known energy balance associated with the 
deformation process (Chrysochoos, 1985; Chrysochoos et al., 2009) then directly gives the dissipated energy ratio.

In addition, the second principle of thermodynamics states that the intrinsic dissipation must be positive in any deformation process (Germain et al., 1983). Then, under adiabatic conditions, the temperature of the material can only rise if the dissipation is the sole heat source.

For the flow rule given by Johnson-Cook's rheological equation, the flow stress, as well as the viscoplastic energy rate and dissipation tend toward zero as the temperature approaches the melting temperature. When tested at a high strain rate, the temperature in the band must then tend asymptotically and monotonically toward this limit melting temperature. Many experimental studies have been conducted to study temperature variations within ASBs. Given the very short-term development of these bands and their very low thickness, pyrometry measurement techniques have been the most commonly used (Hartley et al., 1987; Marchand and Duffy, 1988; Duffy and Chi, 1992; Liao and Duffy, 1998; Ranc et al., 2008; Rittel and Wang, 2008). Continuous improvement of spatial and temporal resolutions of sensors have allowed more reliable temperature measurements. Various studies have shown temperatures of up to $1000^{\circ} \mathrm{C}$ in the shear bands for some materials. However, these temperatures remain well below the melting temperature. These results seem to disagree with the theoretical statement put forward previously and lead to the conclusion that interactions between mechanical and thermal effects are not limited to competition between mechanical dissipation and thermal softening but instead involve another mechanism. In the literature, temperature variations in ASBs are, to our knowledge, in most cases attributed to mechanical dissipation. Some recent studies (Osvski et al., 2012, 2013) highlighted the fact that, depending on the material, some microstructural mechanisms (twinning, dynamic recrystallization) trigger the onset and development of ASBs, while for some other materials, thermal softening remains the main cause. In these papers, it is also claimed that recrystallization enthalpy has no effect on the material response (cf. Fig. 7 in Osvski et al. (2012)). However, Johnson-Cook's rheological equation is highly dependent on the strain-induced temperature and should thus generate another heat source reflecting this strong thermomechanical coupling mechanism. It seems that this source has often been ignored or implicitly neglected. However, is this attitude always well founded? And if not, how could this additional thermomechanical coupling source be estimated in the case of Johnson-Cook's thermoviscoplastic flow?

This paper aims to provide some answers to these two questions. First, Johnson-Cook law is reformulated so that it can be integrated into the framework of the thermodynamics of irreversible processes with internal state variables. The formalism of the generalized standard materials (GSM, Halphen and Son Nguyen, 1975) was chosen to unambiguously define the dissipative and coupling sources associated with the transformation. It should be mentioned here that the integration of Johnson-Cook law in a thermomechanical framework has also been recently achieved by Su (2012) in his Ph.D. thesis, with the aim being to include such a model in a general variational approach based on energy considerations. Note however that the selected state variables and potentials are quite different.

In a second step, the reformulated Johnson-Cook's model is numerically implemented to perform simulations of monotonic tests on the basis of which the complete energy balance is presented and commented. Finally, the mechanical and thermal responses also derived from the model are matched against some experimental results taken from the literature.

\section{Johnson-Cook's flow stress}

\subsection{Reminder}

In the case of one-dimensional monotonous loading, the Johnson-Cook rheological equation assumes that the flow stress $\sigma_{y}$ is a function of the cumulated viscoplastic strain $\left.p=\int_{0}^{t}\left|\dot{\varepsilon}_{v p}(\tau)\right|\right) \mathrm{d} \tau$, of the viscoplastic strain rate $\dot{\varepsilon}_{v p}$, and of the material temperature $T$.

$\sigma_{y}\left(p, \dot{\varepsilon}_{v p}, T\right)=\left(A+B p^{n}\right) f_{d}\left(\dot{\varepsilon}_{v p}\right) f_{t h}(T)$

where $f_{d}$ and $f_{\text {th }}$ are functions that allow us to respectively take the viscous hardening and thermal softening into account.

The first term of the rheological equation describes the monotonous hardening induced by isotropic plastic hardening.

For $\left|\dot{\varepsilon}_{v p}\right|>\dot{\varepsilon}_{0}$, the function $f_{d}$ reads:

$f_{d}\left(\dot{\varepsilon}_{v p}\right)=1+C \ln \left(\frac{\left|\dot{\varepsilon}_{v p}\right|}{\dot{\varepsilon}_{0}}\right)=1+C \ln \left|\dot{\varepsilon}^{*}\right|$.

If $\left|\dot{\varepsilon}_{v p}\right| \leqslant \dot{\varepsilon}_{0}$, Johnson and Cook suppose that the viscoplastic strain rate has no influence on flow stress $\sigma_{y}$ and consequently $f_{d}=1$. At this stage, they also introduce the adimensionnal viscoplastic strain rate defined by $\dot{\varepsilon}^{*}=\frac{\dot{\varepsilon}_{v p}}{\dot{\varepsilon}_{0}}$.

In the same way, for temperatures between the socalled transition temperature named $T_{t}$ and the melting temperature $T_{m}$, they define the function reflecting the thermal softening $f_{t h}$ as follows:

$f_{t h}(T)=1-\left(\frac{T-T_{t}}{T_{m}-T_{t}}\right)^{m}=1-\theta^{* m}$,

where $\theta^{*}$ is an adimensional temperature. If the temperature is less than the transition temperature $T_{t}, \theta^{*} \leqslant 0$, the authors suppose that no thermal softening occurs and then $f_{t h}=1$. At the other end of the range, if the temperature is greater than the melting temperature $T_{m}$, i.e. $\theta^{*} \geqslant 1$, the flow stress vanishes and then $f_{\text {th }}=0$.

The parameters $A, B, C, n$ and $m$ are constants characterizing the studied material. In the pioneering paper of Johnson and Cook (1983), data are given for a wide number of metallic alloys thereby showing the empirical but certainly convenient and efficient character of this behavioral equation. 


\subsection{Rheological equation analysis}

Several hypotheses are formulated to include JohnsonCook's rheological equation within the GSM framework. First, the overall strain $\varepsilon$ is generally assumed to be split into elastic strain $\varepsilon_{e}$ and viscoplastic strain $\varepsilon_{v p}$. This reads:

$\varepsilon=\varepsilon_{e}+\varepsilon_{v p}$.

It is worth noting that this partition does not exist in the original formulation, surely because of the smallness of the elastic strain within ASB as compared to the viscoplastic strain. The elastic strain is introduced here to be able to define the elastic domain and then its boundary where the viscoplasticity develops in accordance with the flow rule defined by Johnson-Cook's equation. The elastic part of the behavior is assumed to be linear, characterized by a constant Young's modulus denoted by $E$. This assumption is certainly questionable in the case of high temperature and/or large deformations. This assumption is adopted hereafter for obvious simplicity reasons.

The viscoplastic part of the behavior is interpreted as being due to the parallel association of viscous and plastic hardening effects. From a rheological standpoint, the viscosity will be represented by a non-linear dashpot and the plasticity by a plastic block whose yield stress depends on the hardening state (Fig. 1).

If $\sigma_{v}$ and $\sigma_{h}$ are the viscous and plastic stresses respectively developed by the dashpot and the plastic block, we then get:

$\sigma=\sigma_{v}+\sigma_{h}$

From the rheological Eq. 1, the viscous stress can be defined by the following nonlinear relation:

$\sigma_{v}= \begin{cases}\mu(p, T) \ln \left(\frac{\left|\dot{\varepsilon}_{v p}\right|}{\dot{\varepsilon}_{0}}\right) \operatorname{sgn}\left(\dot{\varepsilon}_{v p}\right) & \text { if }\left|\dot{\varepsilon}_{v p}\right|>\dot{\varepsilon}_{0}, \\ 0 & \text { if }\left|\dot{\varepsilon}_{v p}\right| \leqslant \dot{\varepsilon}_{0} .\end{cases}$

with $\mu(p, T)=C\left(A+B p^{n}\right)\left(1-\left(\theta^{*}(T)\right)^{m}\right)$. The stress $\sigma_{h}$ can be expressed as a function of the yield stress of the plastic block since viscoplastic flow occurs:

$\sigma_{h}= \begin{cases}\sigma_{0} & \text { if } \dot{\varepsilon}_{v p}>0, \\ -\sigma_{0} & \text { if } \dot{\varepsilon}_{v p}<0 .\end{cases}$

with

$\sigma_{0}=\left(A+B p^{n}\right)\left(1-\left(\theta^{*}(T)\right)^{m}\right)$

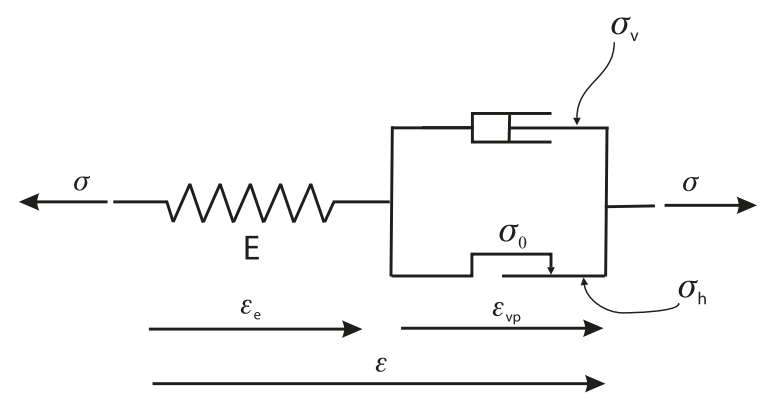

Fig. 1. Elasto-viscoplastic model.
If the viscoplastic strain rate is zero $\left(\dot{\varepsilon}_{v p}=0\right)$, the material state remains within the elastic domain characterized by $\left|\sigma_{h}\right| \leqslant \sigma_{0}$. This one-dimensional elastic domain is then usually defined using the yield function $f_{h}$ that can be expressed as:

$f_{h}\left(\sigma_{h}\right)=\left|\sigma_{h}\right|-\sigma_{0}$.

The function $f_{h}$ is negative within the elastic domain. For monotonous viscoplastic loading, the overall stress developed by the dashpot and the plastic block is then equal to the viscoplastic flow stress proposed by Johnson and Cook:

$$
\begin{aligned}
\sigma_{y} & =\sigma_{h}+\sigma_{v} \\
& =\left(A+B p^{n}\right)\left(1-\left(\theta^{*}(T)\right)^{m}\right)+\mu(p, T) \ln \left(\frac{\dot{\varepsilon}_{v p}}{\dot{\varepsilon}_{0}}\right)
\end{aligned}
$$

\section{Thermodynamic framework}

The goal of what follows is to include Johnson-Cook's rheological equation in the convenient GSM framework (Halphen and Son Nguyen, 1975).

\subsection{State variables and free energy}

Four state variables are chosen to define the material state: the temperature $T$, the overall strain $\varepsilon$, the viscoplastic strain $\varepsilon_{v p}$ and the viscoplastic cumulated strain $p$ which allows us to consider the monotonous evolution of the hardening state (isotropic hardening).

As for Prager's and Prandtl-Reuss's models, the plastic hardening energy is supposed to be stored within the material microstructure. This nondissipated part of the anelastic deformation energy is the so-called stored energy. The volume free energy can then be expressed as the sum of the elastic strain energy, the stored or released heat induced by temperature variations and the stored energy in the plastic hardening:

$$
\begin{aligned}
\psi\left(T, \varepsilon, \varepsilon_{v p}, p\right)= & \underbrace{\frac{1}{2} E\left(\varepsilon-\varepsilon_{v p}\right)^{2}}_{\text {Elastic energy }}-\underbrace{\rho C_{p}\left(T \ln \frac{T}{T_{a}}-T\right)}_{\text {Calorific energy }} \\
& +\underbrace{\frac{B}{n+1} p^{n+1}\left(1-\left(\theta^{*}(T)\right)^{m}\right)}_{\text {Mechanical stored energy }}
\end{aligned}
$$

with $T_{a}$ representing the room temperature, $\rho$ the mass density and $C_{p}$ the specific heat.

Each state variable is associated with a conjugate variable defined as the partial derivative of the free energy with respect to the state variable. In the case where the free energy has the form given by Eq. (11), the following state equations can be derived:

$$
\left\{\begin{array}{l}
\frac{\partial \psi}{\partial T}=-\rho C_{p} \ln \frac{T}{T_{a}}-\frac{m B}{n+1} p^{n+1} \frac{\theta^{* m-1}}{T_{m}-T_{t}}=-\rho S \\
\frac{\partial \psi}{\partial \varepsilon}=E\left(\varepsilon-\varepsilon_{v p}\right)=\sigma_{r} \\
\frac{\partial \psi}{\partial \varepsilon_{v p}}=-E\left(\varepsilon-\varepsilon_{v p}\right)=-\sigma_{r}=A_{\varepsilon_{v p}} \\
\frac{\partial \psi}{\partial p}=B p^{n}\left(1-\theta^{* m}\right)=A_{p}
\end{array}\right.
$$


where $s$ stands for the volume entropy, $\sigma_{r}$ the reversible stress and $A_{\varepsilon_{v p}}, A_{p}$ the conjugate variables of the state variables $\epsilon_{v p}$ and $p$, respectively. In the state equation defining the entropy, it is worth noting that the last term is associated with the stored energy, which depends on the temperature because of the thermal softening.

\subsection{Second principle, flux and dissipation potential}

The local expression of the second law of thermodynamics, often called the Clausius-Duhem inequality (Germain et al., 1983), expresses the positivity of the total dissipation denoted by $d_{\text {tot }}$. This dissipation is split into two terms, i.e. the intrinsic dissipation $d_{1}$ and the thermal dissipation $d_{2}$, assumed to be separately positive:

$d_{\text {tot }}=d_{1}+d_{2}$.

The thermal dissipation is defined by:

$d_{2}=-\frac{\vec{q}}{T} \cdot \operatorname{grad} T \geqslant 0$

where $\vec{q}$ is the heat influx vector. The intrinsic dissipation can then here be written as:

$d_{1}=\sigma \dot{\varepsilon}-\frac{\partial \psi}{\partial \varepsilon} \dot{\varepsilon}-\frac{\partial \psi}{\partial \varepsilon_{v p}} \dot{\varepsilon}_{v p}-\frac{\partial \psi}{\partial p} \dot{p}$

$=\left(\sigma-\sigma_{r}\right) \dot{\varepsilon}-A_{\varepsilon_{v p}} \dot{\varepsilon}_{v p}-A_{p} \dot{p} \geqslant 0$

The thermal dissipation can be seen as the product of a thermodynamic force $-\frac{\text { grad } T}{T}$ by the heat influx $\vec{q}$. In the same way, the intrinsic dissipation can be written as:

$d_{1}=X_{i} \dot{x}_{i}$

where $\dot{x}_{i}$ stands for the fluxes (rates) of the state variables and $X_{i}$ the associated thermodynamic forces.

The state variable fluxes are here $\dot{\varepsilon}, \dot{\varepsilon}_{v p}$ and $\dot{p}$ while $X_{\dot{\varepsilon}}, X_{\dot{\varepsilon}_{v p}}$ and $X_{\dot{p}}$ are the associated thermodynamic forces. The irreversible stress $\sigma_{i r}$ is conventionally defined as:

$\sigma_{i r}=X_{\dot{\varepsilon}}$

in order to get:

$\sigma=\sigma_{r}+\sigma_{i r}$

Complementary equations are required to complete the description of the material behavior. They specify the nature of irreversibility accompanying the deformation process. This description is usually made through the correspondence between fluxes and thermodynamic forces. In the GSM framework, the existence of a convex dissipation potential function of the state variable rates $\varphi\left(\dot{\varepsilon}, \dot{\varepsilon}_{v p}, \dot{p} ; T, p\right)$ is assumed. This potential is positive and minimal at $\dot{\varepsilon}=0, \dot{\varepsilon}_{v p}=0$ and $\dot{p}=0$ and is such that:

$X_{\dot{\varepsilon}}=\frac{\partial \varphi}{\partial \dot{\varepsilon}}=\sigma_{i r}, \quad X_{\dot{\varepsilon}_{v p}}=\frac{\partial \varphi}{\partial \dot{\varepsilon}_{v p}} \quad$ and $\quad X_{\dot{p}}=\frac{\partial \varphi}{\partial \dot{p}}$

The convexity properties imposed on the dissipation potential ensure the positivity of the dissipation whatever the thermodynamic process. As for the state potentials, other dissipation potentials can be proposed in order to use a more appropriate set of descriptive variables. In viscoplasticity, it can be convenient to formulate the dissi- pation potentiel as a function of the thermodynamic forces. This is the dual dissipation potential $\varphi^{*}\left(X_{\dot{\varepsilon}}, X_{\dot{\varepsilon}_{v p}}, X_{\dot{p}}\right)$ from which the state variable rates are derived:

$\dot{\varepsilon}=\frac{\partial \varphi^{*}}{\partial X_{\dot{\varepsilon}}} \quad \dot{\varepsilon}_{v p}=\frac{\partial \varphi^{*}}{\partial X_{\dot{\varepsilon}_{v p}}} \quad$ and $\quad \dot{p}=\frac{\partial \varphi^{*}}{\partial X_{\dot{p}}}$

The dual dissipation potential $\varphi^{*}$ is obtained using the Legendre-Fenchel transform of $\varphi$, which holds for differentiable or sub-differentiable potentials, as is the case in plasticity (Moreau, 1962).

\subsection{Complementary hypotheses}

The first hypothesis assumes that the intrinsic dissipation is only generated by the viscoplastic mechanisms and then only by $\dot{\varepsilon}_{v p}$ and $\dot{p}$. This means that no irreversibility is associated with $\dot{\varepsilon}$. Consequently

$\frac{\partial \varphi}{\partial \dot{\varepsilon}}=\sigma_{i r}=0$

and then, given that

$\sigma_{i r}=\sigma-\sigma_{r}$,

this leads to

$\sigma=\sigma_{r}=\frac{\partial \psi}{\partial \varepsilon}$

For such conditions, the intrinsic dissipation can be written as:

$d_{1}=\sigma \dot{\varepsilon}_{v p}-A_{p} \dot{p}=X_{\dot{\varepsilon}_{v p}} \dot{\varepsilon}_{v p}+X_{\dot{p}} \dot{p}$

And then finally:

$X_{\dot{\varepsilon}}=0, \quad X_{\dot{\varepsilon}_{v p}}=\sigma, \quad X_{\dot{p}}=-A_{p}=-B p^{n}\left(1-\theta^{*}(T)^{m}\right)$.

The dual dissipation to determine is then a function of the two thermodynamic forces: $X_{\dot{\varepsilon}_{v p}}$ and $X_{\dot{p}}$.

The second hypothesis resumes the parallel construction of the viscous and plastic irreversibilities in order to separately identify the dual dissipation potential of both rheological elements: the plastic block and the nonlinear dashpot.

Regarding the plastic block, the dual dissipation potential is the indicator function of the convex elasticity domain, here defined by $f_{h}\left(\sigma_{h}\right)=\left|\sigma_{h}\right|-\sigma_{0}$. The dual dissipation potential $\mathcal{I}_{f_{h}}$ is then defined by:

$\mathcal{I}_{f_{h} \leqslant 0}=\varphi_{h}^{*}\left(\sigma_{h}\right)= \begin{cases}0 & \text { if } f_{h} \leqslant 0 \\ +\infty & \text { if } f_{h}>0\end{cases}$

The plastic strain rate is related to the subdifferential of $\mathcal{I}_{f_{h}}$ :

$\dot{\varepsilon}_{p} \in \partial \mathcal{I}_{f_{h} \leqslant 0}$.

Note here that the subset $\partial \mathcal{I}_{f_{h} \leqslant 0}(x)$ is the sub-differential in $x$ of the indicator function $\mathcal{I}_{f_{h} \leqslant 0}(x)$. If $x$ is inside the convex domain $f_{h} \leqslant 0, \partial \mathcal{I}_{f_{h} \leqslant 0}(x)$ is reduced to $\{0\}$, and if $x$ is on the boundary $f_{h}(x)=0, \partial \mathcal{I}_{f_{h} \leqslant 0}(x)$ is the normal cone to the set $f_{h} \leqslant 0$, (see Fig. 2 ). The elasticity domain size changes here not only with the hardening state progress but also with the material temperature. The dual dissipation potentiel then depends on the thermodynamic force $\sigma_{h}$, but also 


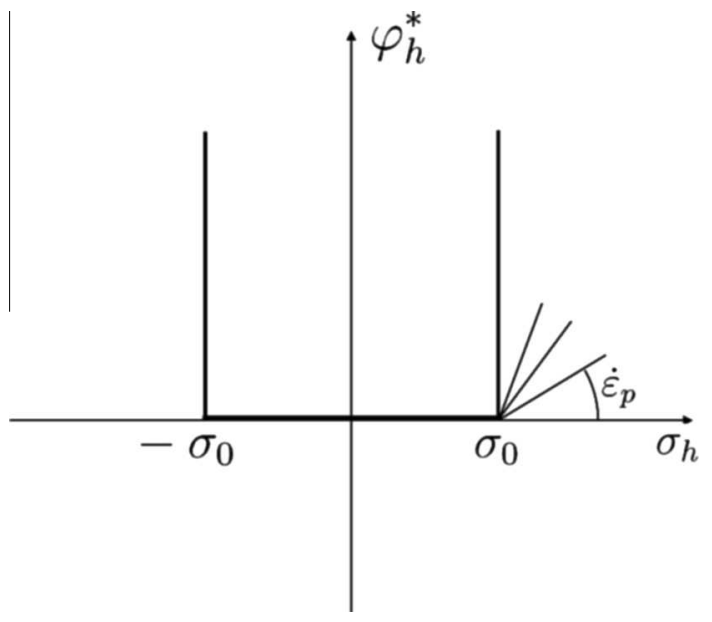

Fig. 2. Dual dissipation potential of the plastic block of yield stress $\sigma_{0}$.

must involve the state variables $p$ and $T$ which act as parameters characterizing the size of the elasticity domain.

The dual dissipation potential of the nonlinear dashpot can be obtained while considering the following evolution equation:

$\dot{\varepsilon}_{v}=\frac{\partial \varphi_{v}^{*}}{\partial \sigma_{v}}$

The inversion of the Eq. 6 leads to:

$\dot{\varepsilon}_{v}=\dot{\varepsilon}_{0} \exp \left(\frac{\left|\sigma_{v}\right|}{\mu(p, T)}\right) \operatorname{sgn}\left(\sigma_{v}\right)$

After integration, we obtain:

$\varphi_{v}^{*}\left(\sigma_{v} ; p, T\right)=\mu(p, T) \dot{\varepsilon}_{0}\left(\exp \left(\frac{\left|\sigma_{v}\right|}{\mu(p, T)}\right)-1\right)$

where the integration constant has been chosen to get $\varphi_{v}^{*}(0)=0$.

Both dual dissipation potentials $\varphi_{v}^{*}$ et $\varphi_{h}^{*}$ are plotted in Figs. 2 and 3.

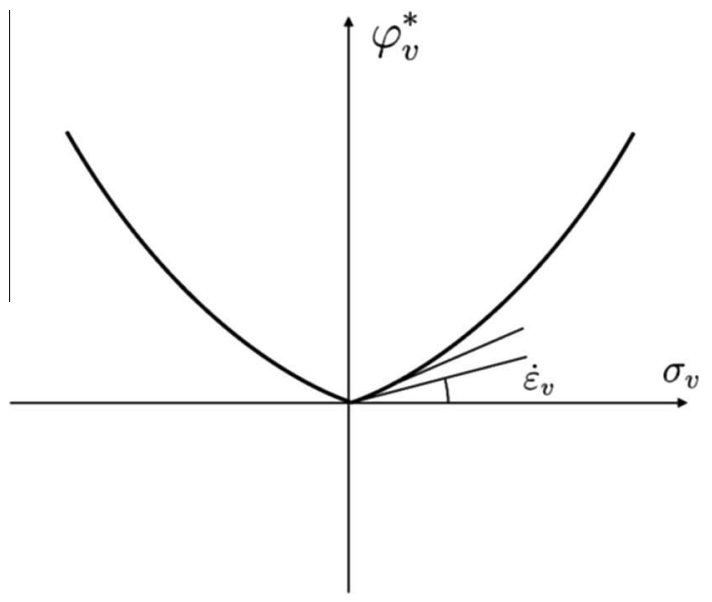

Fig. 3. Dual dissipation potential associated with nonlinear dashpot.
The paralleling of plastic and viscous irreversibilities (i.e., $\dot{\varepsilon}_{v}=\dot{\varepsilon}_{p}=\dot{\varepsilon}_{v p}, \sigma=\sigma_{h}+\sigma_{v}$ ) implies that the overall dual dissipation potential $\varphi^{*}$ can be derived from the infconvolution of $\varphi_{h}^{*}$ and $\varphi_{v}^{*}$ defined by Moreau (1963):

$\left(\varphi_{h}^{*} \star_{\text {inf }} \varphi_{v}^{*}\right)(\sigma ; p, T)=\inf _{\sigma^{\prime}}\left\{\varphi_{h}^{*}\left(\sigma-\sigma^{\prime} ; p, T\right)+\varphi_{v}^{*}\left(\sigma^{\prime} ; p, T\right)\right\}$.

The dual potential becomes:

$\varphi^{*}(\sigma ; p, T)=\mu(p, T) \dot{\varepsilon}_{0}\left(\exp \left(\frac{\left(|\sigma|-\sigma_{0}\right)^{+}}{\mu(p, T)}\right)-1\right)$

where $(.)^{+}$is the positive part of (.).

Before deriving the complementary constitutive equations, the dual dissipation potential must be rewritten as a function of the thermodynamic forces of the model, as defined in (Eq. (26)). Noting that the difference $|\sigma|-\sigma_{0}$ can be written as:

$|\sigma|-\sigma_{0}=\left|X_{\dot{\varepsilon}_{v p}}\right|+X_{\dot{p}}-A\left(1-\theta^{*}(T)^{m}\right)=f_{v p}$,

the dual dissipation potential takes the following final form:

$\varphi^{*}\left(X_{\dot{\varepsilon}_{v p}}, X_{\dot{p}} ; p, T\right)=\mu(p, T) \dot{\varepsilon}_{0}\left(\exp \left(\frac{\left(\left|X_{\dot{\varepsilon}_{v p}}\right|+X_{\dot{p}}-A\left(1-\theta^{*}(T)^{m}\right)\right)^{+}}{\mu(p, T)}\right)-1\right)$.

Fig. 4 shows the graph of the dual dissipation potential as a function of $\sigma=X_{\dot{\varepsilon}_{v p}}$ for fixed values of $X_{\dot{p}}$ and $T$.

\subsection{Evolution equations}

The evolution equations read:

$\left\{\begin{array}{l}\sigma_{i r}=0 \\ \dot{\varepsilon}_{v p}=\frac{\partial \varphi^{*}}{\partial X_{\dot{\varepsilon}_{v p}}}=\dot{\varepsilon}_{0} \exp \left(\frac{\left(f_{v p}\right)^{+}}{\mu(p, T)}\right) \operatorname{sgn} \sigma \\ \dot{p}=\frac{\partial \varphi^{*}}{\partial X_{\dot{p}}}=\dot{\varepsilon}_{0} \exp \left(\frac{\left(f_{v p}\right)^{+}}{\mu(p, T)}\right)=\left|\dot{\varepsilon}_{v p}\right|\end{array}\right.$

Associated with the state equations, these evolution equations complete the set of behavioral constitutive equations.

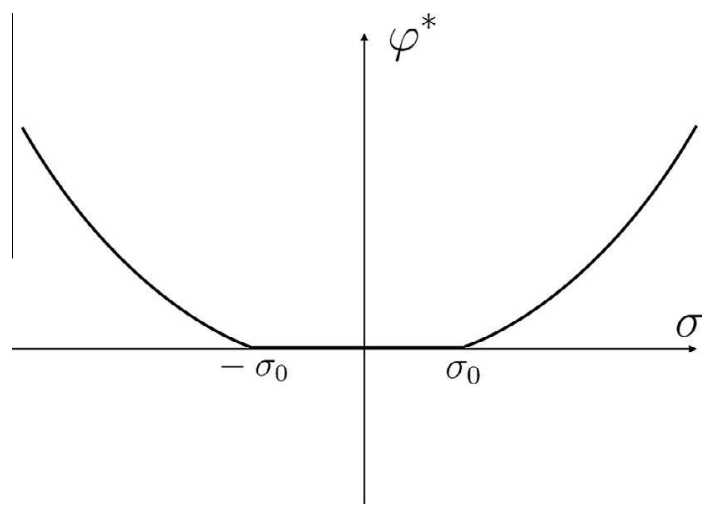

Fig. 4. Graph of the dual dissipation potential at given $X_{\dot{p}}$ and $T$. 


\subsection{Heat equation}

The combination of local expressions of the first and second principles of thermodynamics can be used to derive the heat diffusion equation (Germain et al., 1983). In the adiabatic case, the heat equation is here written as:

$\rho C_{p} \dot{T}=\underbrace{X_{\dot{\varepsilon}_{v p}} \dot{\varepsilon}_{v p}+X_{\dot{p}} \dot{p}}_{d_{1}}+\underbrace{T \frac{\partial^{2} \psi}{\partial T \partial p} \dot{p}}_{s_{\text {thm }}}$

with $d_{1}$ being the intrinsic dissipation and $s_{\text {thm }}$ the heat source associated with thermoplastic coupling. After developing and replacing the different source terms, the heat equation becomes:

$\rho C_{p} \dot{T}=\sigma \dot{\varepsilon}_{v p}-B p^{n}\left(1-\left(\theta^{*}\right)^{m}\right) \dot{p}-m \frac{T}{T_{m}-T_{a}}\left(\theta^{*}\right)^{m-1} \dot{p}$

From Eq. $36\left(\left|\dot{\varepsilon}_{v p}\right|=\dot{p}\right)$ we obtain:

$\rho C_{p} \dot{T}=\left\{|\sigma|-B p^{n}\left[\left(1-\left(\theta^{*}\right)^{m}\right)-m \frac{T}{T_{m}-T_{a}}\left(\theta^{*}\right)^{m-1}\right]\right\} \dot{p}$

\section{Numerical applications of the model}

Two materials were selected to analyze the effects induced by the coexistence of dissipation and a thermomechanical source of coupling: the titanium alloy TA6V and the low structural steel alloy HY100. The parameters of the Johnson-Cook flow rule are respectively given by Ranc et al. (2006) and Ranc (2004) for TA6V and are identified from the torsion tests carried out by Marchand and Duffy (1988) for HY100 steel. The identification method is detailed in Ranc et al. (2006) and Ranc (2004). This identification is done using the stress-strain responses of materials associated with monotone loading performed at different strain rates and environmental temperatures. It is worth noting that the self-heating of the specimens is, to our knowledge, always neglected. All of the parameters as well as the physical properties of the two materials (mass density $\rho$, specific heat $C_{p}$ and shear modulus $G$ ) are given in the Table 1 . A total strain rate of $1000 s^{-1}$ is imposed.

In order to view the effects of thermoplastic coupling on the thermal response of ASBs, numerical simulations integrating the above model were conducted. For a given tem-

Table 1

Parameters of Johnson-Cook's law for TA6V and HY100.

\begin{tabular}{lll}
\hline Parameter & TA6V & HY100 \\
\hline$A$ & $500 \mathrm{MPa}$ & $300 \mathrm{MPa}$ \\
$B$ & $160 \mathrm{MPa}$ & $250 \mathrm{MPa}$ \\
$n$ & 0.25 & 0.18 \\
$C$ & 0.008 & 0.011 \\
$\dot{\gamma}_{0}=\sqrt{3} \dot{\varepsilon}_{0}$ & $0.1 \mathrm{~s}^{-1}$ & $10^{-4} \mathrm{~S}^{-1}$ \\
$T_{t}$ & $20^{\circ} \mathrm{C}$ & $20^{\circ} \mathrm{C}$ \\
$T_{m}$ & $1670^{\circ} \mathrm{C}$ & $1500^{\circ} \mathrm{C}$ \\
$m$ & 1,055 & 1.55 \\
$\rho$ & $4400 \mathrm{kgm}^{-3}$ & $7860 \mathrm{kgm}^{-3}$ \\
$C_{p}$ & $656 \mathrm{Jkg}^{-1} \mathrm{~K}^{-1}$ & $473 \mathrm{Jg}^{-1} \mathrm{~K}^{-1}$ \\
$G$ & $39 \mathrm{GPa}$ & $80 \mathrm{GPa}$ \\
\hline
\end{tabular}

perature, the calculation of the elasto-visco-plastic solution is carried out with an implicit method which uses, according to the form of the nonlinear equation to solve, a combination of bisection, secant, and inverse quadratic interpolation methods. The adiabatic assumption considerably simplifies the temperature variation calculation: knowing the heat source from the elasto-visco-plastic solution on two successive steps, the temperature is updated via the differential heat equation (Eq. 39). A simple Euler type explicit integration scheme was used.

Fig. 5 shows variations in the shear stress and temperature according to the total shear strain in the case of the titanium alloy TA6V. The stress increases until a maximum, which corresponds to the moment when the hardening induced by the viscoplastic work hardening is compensated by the thermal softening effect. The corresponding strain, stress and temperature are respectively $0.31,1130 \mathrm{MPa}$ and $117^{\circ} \mathrm{C}$. After this maximum stress, the temperature increases during thermal softening until reaching a maximum of $932^{\circ} \mathrm{C}$ for a strain of about 10.4 . The temperature then peaks when the dissipative and coupling sources are of the same intensity and the opposite sign. Fig. 6 shows the various powers involved:

- the anelastic energy rate: $\sigma \dot{\varepsilon}_{v p}$,

- the stored energy rate by strain hardening $X_{\dot{p}} \dot{p}=B p^{n}\left(1-\left(\theta^{*}\right)^{m}\right) \dot{p}$,

- the thermoplastic coupling source: $-m B \frac{T}{T_{m}-T_{a}}\left(\theta^{*}\right)^{m-1} \dot{p}$,

- the intrinsic dissipation which is the difference between the anelastic energy rate and the stored energy rate.

Taylor-Quinney's coefficient may be rewritten in the following form on the basis of the definition of the various energy rates:

$\beta_{\mathrm{TQ}}=\frac{B p^{n}\left(1-\left(\theta^{*}\right)^{m}\right) \dot{p}}{\sigma \dot{\varepsilon}_{v p}}$

In the same spirit, the coefficient $\beta_{H}$, which is the ratio of the calorific energy rate on the anelastic energy rate, can be defined. Naturally $\beta_{H}=\beta_{T Q}$ as long as the dissipation is the only heat source induced by the strain process. Note that, in the case of a monotonous loading, all of these energy rates are positive except the energy rate associated with thermoplastic coupling, which is negative.

In order to illustrate the importance of the thermoplastic source, figs. 7 and 8 show the temperature and stress patterns during loading while neglecting the coupling source initially and then by taking it into account. As discussed in the introduction, when only the dissipation is taken into account, the temperature can only increase in a monotonous way and asymptotically tend towards the melting point of the material (here $1670^{\circ} \mathrm{C}$ for TA6V).

When the two sources are taken into account, the coupling term very quickly becomes significant. This notably reduces the temperature variation and there is even a temperature decrease as soon as the intensity of the coupling source becomes greater than the dissipation. As also discussed in introduction, the integration of Johnson-Cook's rheological equation within a thermodynamic framework 


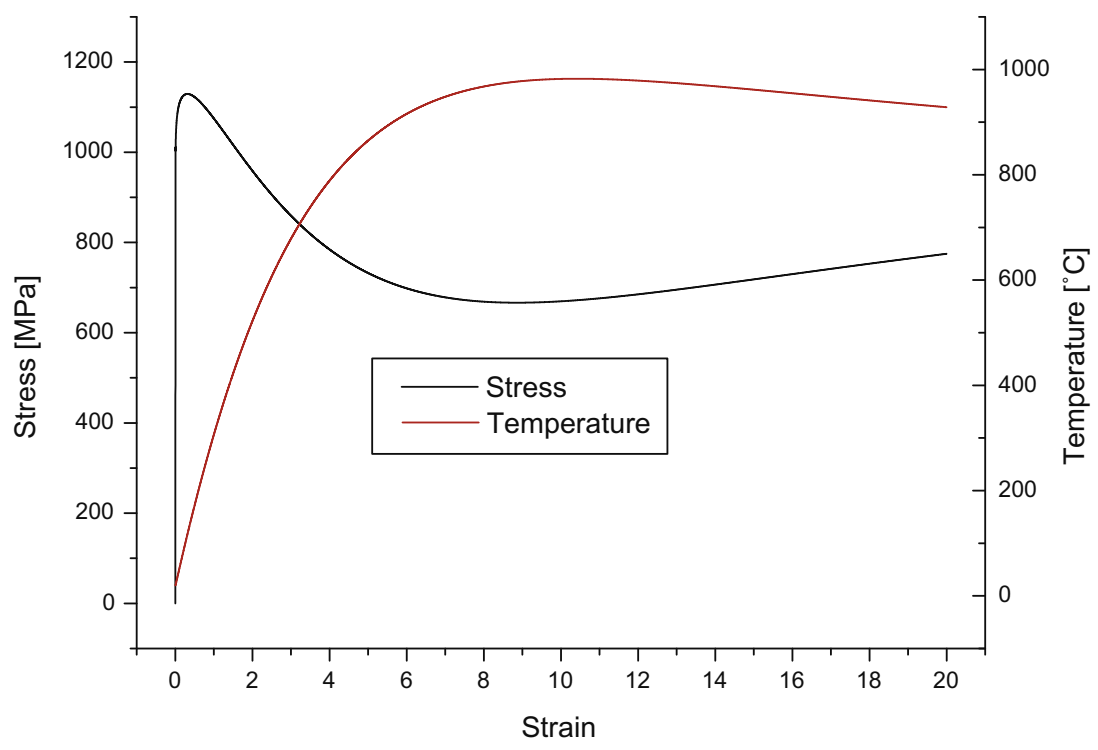

Fig. 5. Variations in stress and temperature according to the strain (Case of TA6V).

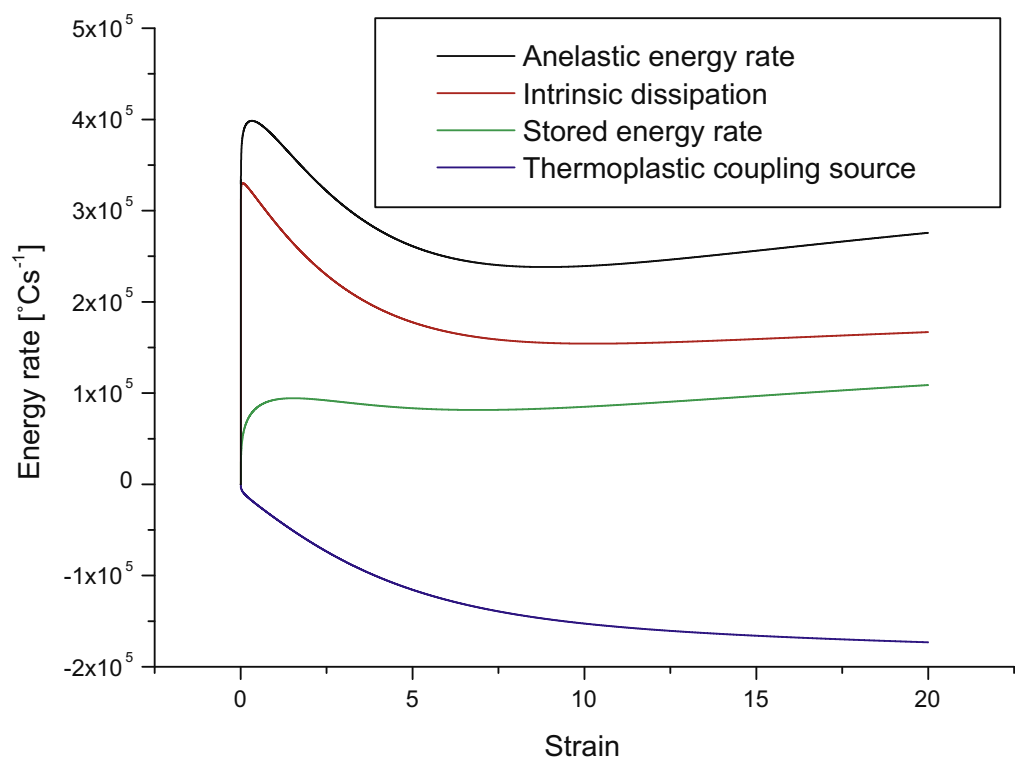

Fig. 6. Variations in the various energy rates (case of TA6V).

enables determination of the evolution of $\beta_{T Q}$ starting from the energy rate balance shown in Fig. 6 .

Fig. 9 shows the evolution of $\beta_{T Q}$ and $\beta_{H}$ according to the total strain. The fact that the dissipative and coupling sources here have opposite signs leads to a rapid divergence in the two coefficients. When the involved calorific energy rate is considered in this paper, the corresponding fraction quickly decreases and even becomes negative as soon as the intensity of the coupling source is higher than the dissipated power. Note that $\beta_{T Q}$ also decreases. This decrease is due to the strong assumption stipulated in paragraph 3.1 that all of the energy rate consumption during work hardening remains stored. This was already dis- cussed in Chrysochoos and Belmahjoub (1992) for quasistatic monotonous tests and in Rittel et al. (2012) for dynamic tests. Other assumptions could have been used. In Bever et al., for example, it is assumed that the stored energy is proportional to the square of the work hardening stress. This assumption basically does not change the focus of this paper which aims to show the importance of coupling mechanisms induced by thermal softening. Naturally, if it is assumed that all the work hardening energy is dissipated, there is no stored energy and thus no coupling source, i.e., heat source representing the variation of the stored energy induced by the temperature. This assumption is of course unrealistic for metallurgical, thermodyn- 


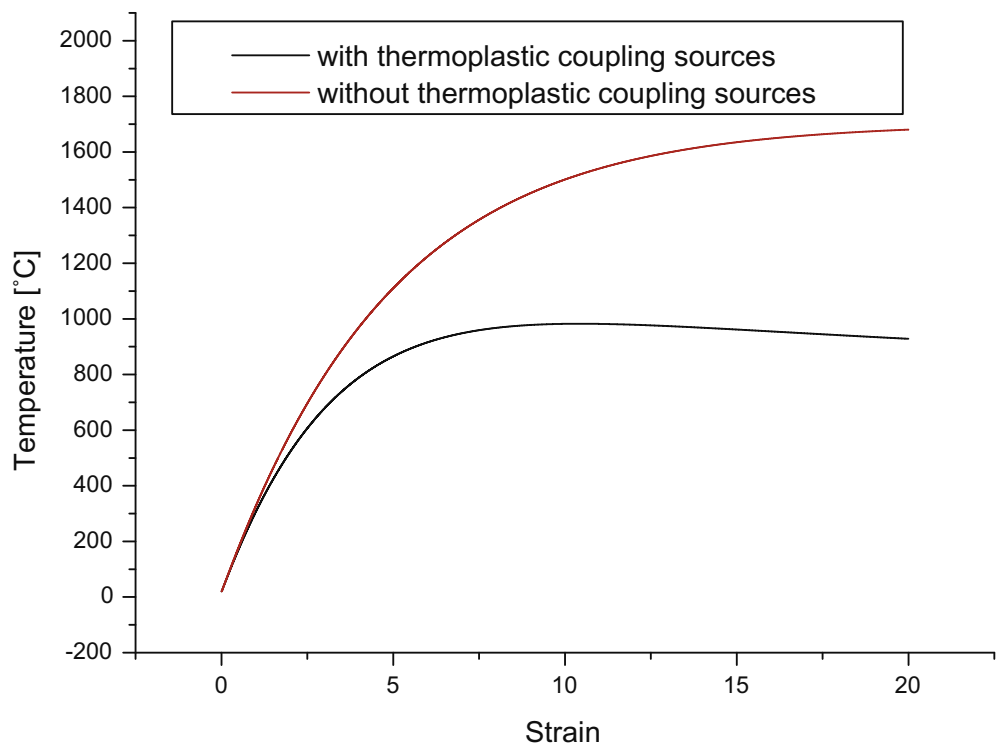

Fig. 7. Effect of thermoplastic coupling on the temperature pattern (TA6V).

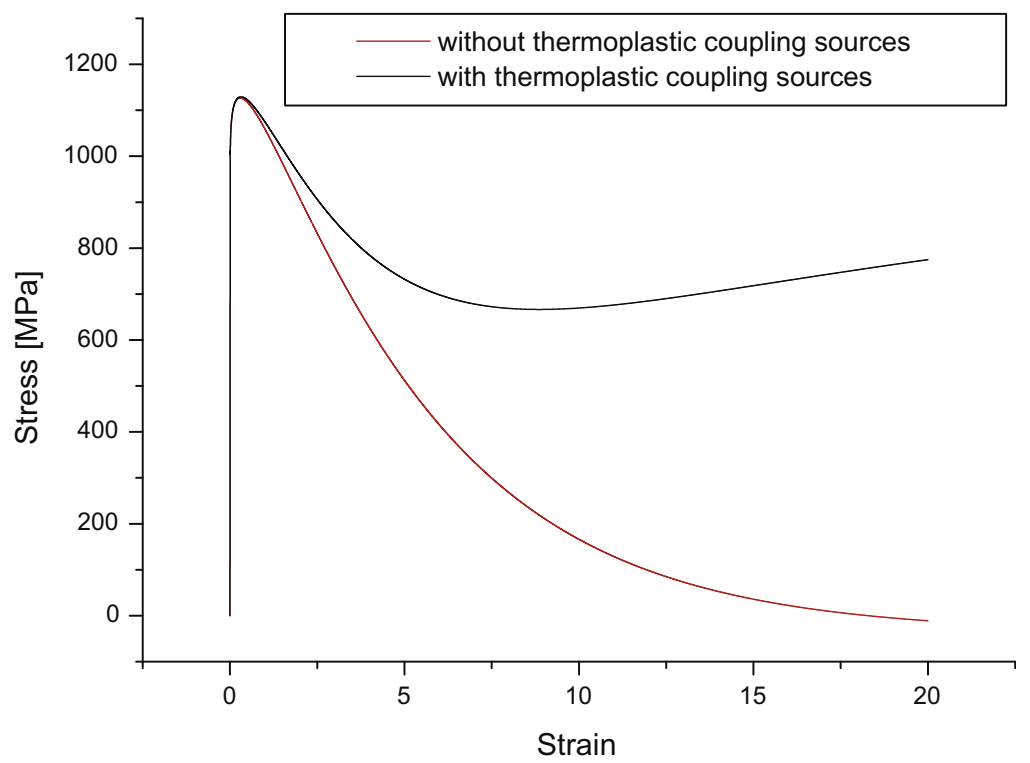

Fig. 8. Effect of thermoplastic coupling on the stress pattern (TA6V).

mic and thermal reasons. From a thermal standpoint, a value of $\beta_{\mathrm{TQ}}=\beta_{H}=1$ would lead to even more significant temperature increases than those shown in Fig. 7 obtained with a $\beta_{T Q}$ coefficient of about 0.7 .

The preceding thermomechanical analysis was also carried out in the case of HY100 steel. Fig. 10 respectively represents variations in stress and temperature according to the total strain. Variations in the energy rate balance are given in Fig. 11. The general appearance of the energy rate balance remains very close to that found in the case of the titanium alloy. The maximum temperature is $750^{\circ} \mathrm{C}$, whereas the melting point is $1500^{\circ} \mathrm{C}$.

\section{Comparison with some experimental results from the literature}

In the case of the TA6V alloy, the temperature measurements in ASBs were carried out by Liao and Duffy (1998) and more recently by Ranc et al. (2008) in the case of torsion tests with Hopkinson bars. Liao and Duffy measured temperature variations with a bar of infrared detectors during formation of an adiabatic shear band. The size of the measurement zone corresponding to one detector is $17 \mu \mathrm{m}$. The experimental results show a very fast increase in the temperature followed by a fast decrease. 


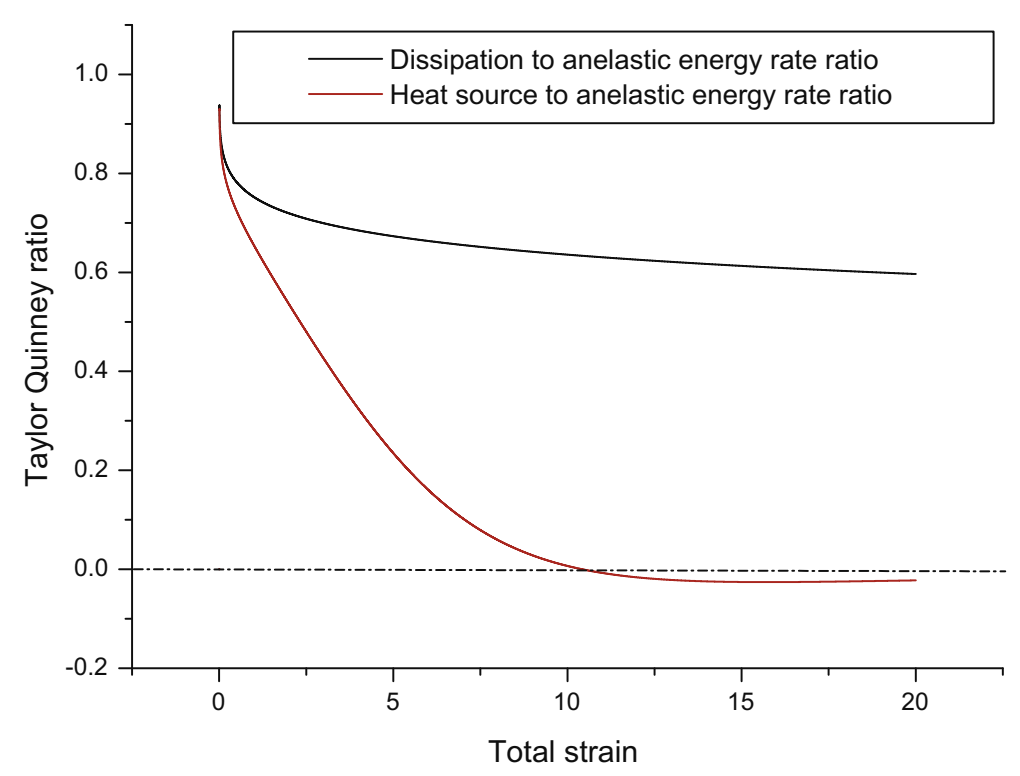

Fig. 9. Variations in Taylor Quinney’s coefficient (TA6V).

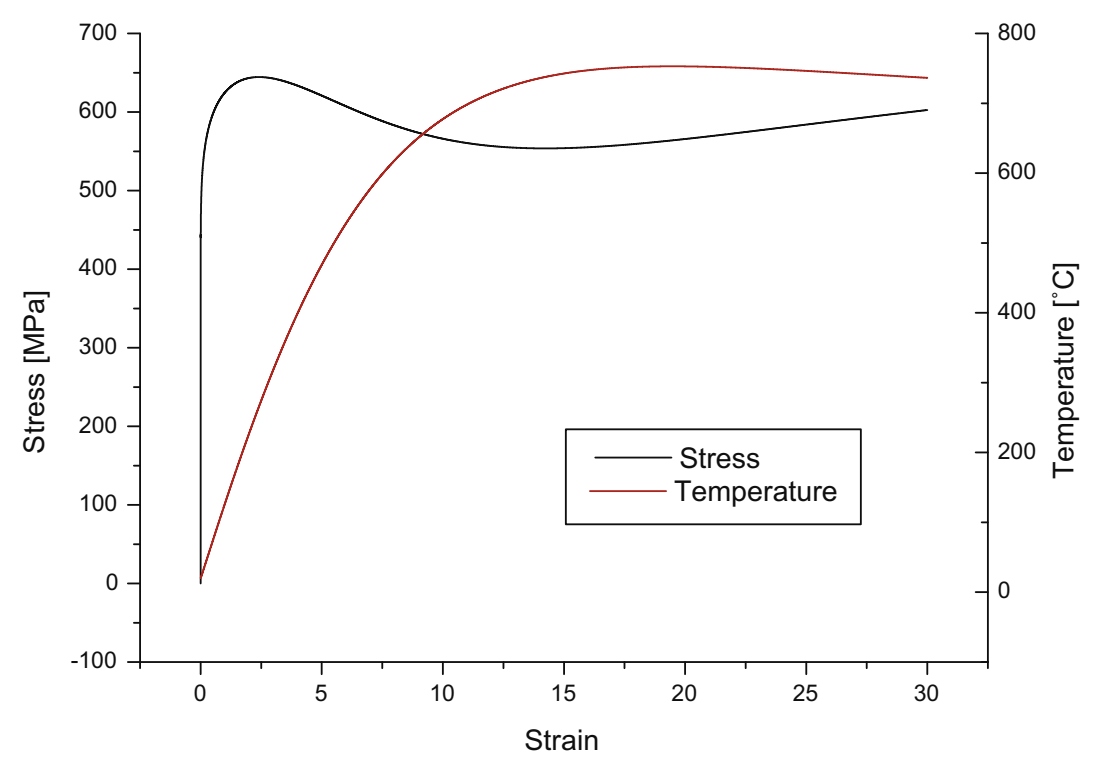

Fig. 10. Variations in stress and temperature according to strain (case of HY100 steel).

The maximum temperature is estimated to be $550^{\circ} \mathrm{C}$. On the other hand, Ranc et al. mapped the temperature in a fully formed adiabatic shear band using a visible camera with a spatial resolution of about $2 \mu \mathrm{m}$. They found a maximum temperature of $1100^{\circ} \mathrm{C}$. The ASB width, which is defined as the width of the zone of intense strain, was estimated by Giovanola to be $10 \mu \mathrm{m}$ in TA6V Giovanola, 1988. The measurements of Liao and Duffy seemed to underestimate the maximum temperature reached at the center of the band because of the spatial resolution of the experimental device, which was higher than the band width.
In both cases, the measured temperatures are much lower than the melting point of the material. As illustrated in Fig. 12, the maximum measured temperature value is in close agreement with the simulations carried out in this study which take the thermoplastic coupling source into account and where the maximum temperature is about $930^{\circ} \mathrm{C}$.

Several assumptions are proposed in the literature to explain the fast temperature decrease. The main explanation concerns the association of the temperature decrease with crack formation and propagation. This crack leads to a decrease in yield stress and thus a reduction in heat 


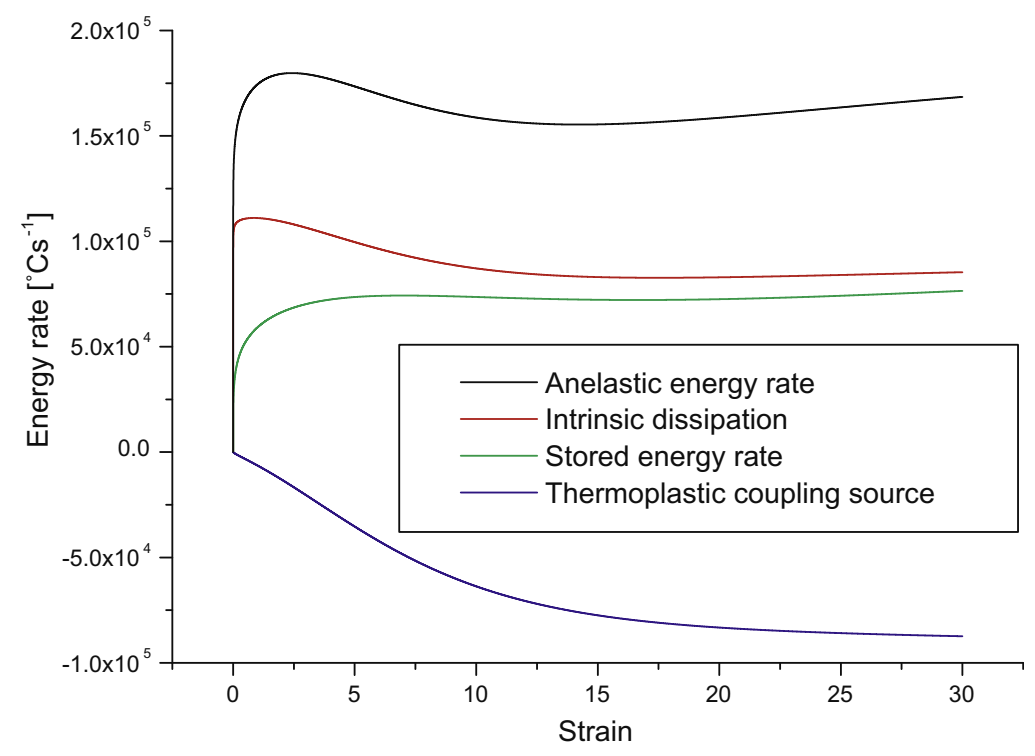

Fig. 11. Variations in the different energy rates (case of HY100 steel).

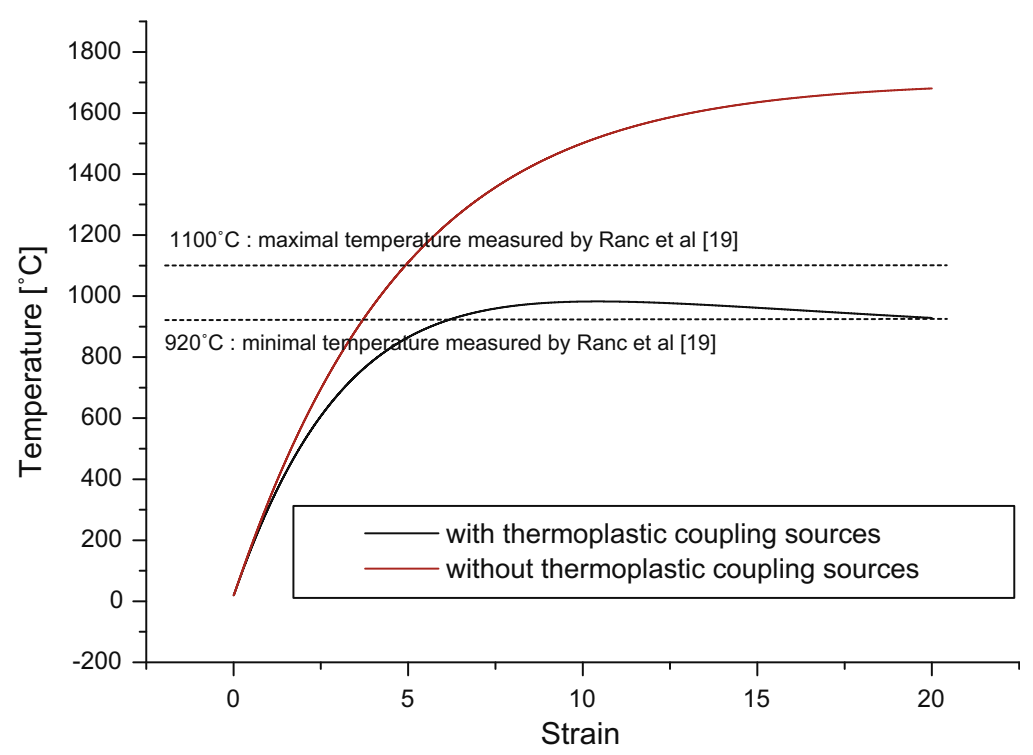

Fig. 12. Comparison of temperature evolution with and without thermoplastic coupling sources with experimental results (case of TA6V).

sources. The cooling in the shear band is then explained by the heat diffusion perpendicular to the direction of the band. A second explanation is given, for instance, by Giovanola (1988): the metallographic observations of the bands generally reveal the presence of microvoids in the ASB. The formation of these microvoids decreases the stress (geometrical softening), thus prompting a decrease in temperature, as in the previous explanation.

In the case of HY100 steel, the maximum temperatures measured by Marchand and Duffy (1988) were between $424^{\circ} \mathrm{C}$ and $590^{\circ} \mathrm{C}$ during a torsion test with Hopkinson bars. For this steel, the bandwidths were about $20 \mu \mathrm{m}$ and $40 \mu \mathrm{m}$, i.e. slightly lower than the size of the temperature measurement area $(35 \mu \mathrm{m})$. The maximum temperatures really reached in the bands must thus be higher. Marchand and Duffy estimated these real temperatures at between $875^{\circ} \mathrm{C}$ and $1140^{\circ} \mathrm{C}$ by taking the real band size into account. Fig. 13 compares the simulated results (computed with and without the thermoplastic coupling sources) with the experimental results of Marchand and Duffy (1988). The maximum temperatures are much lower than the melting point of the material and in close agreement with those calculated by our numerical simulation with the thermoplastic coupling effect. Local strains of about 1900\% were observed before the crack formation. 


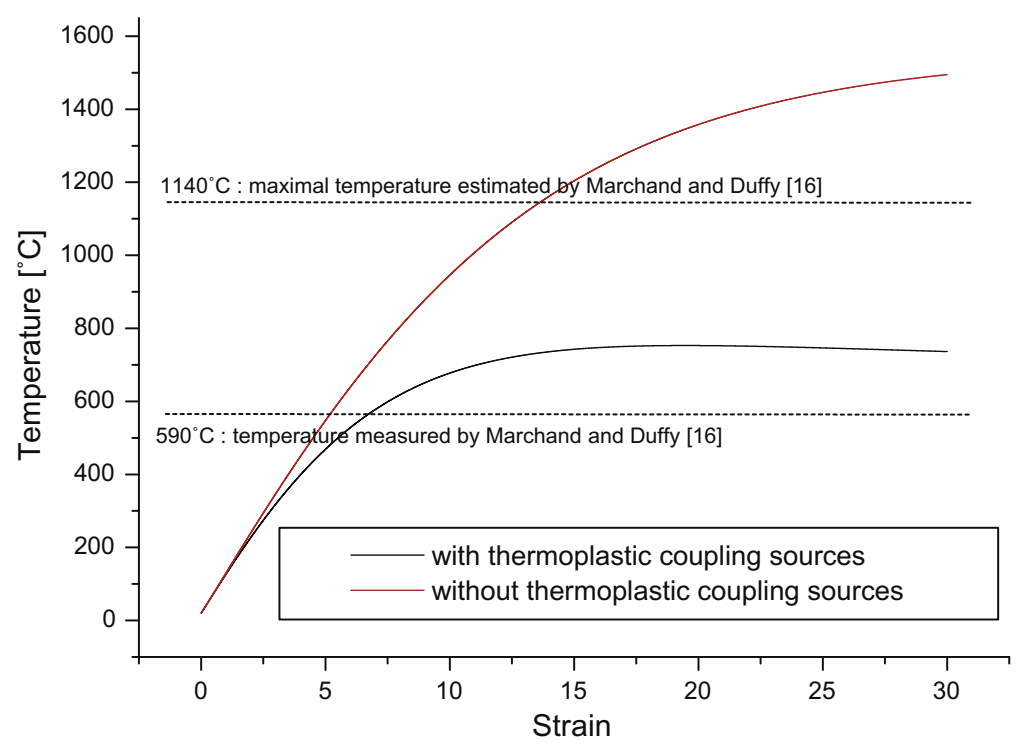

Fig. 13. Comparison of temperature evolution with and without thermoplastic coupling sources with experimental results (case of HY100).

\section{Conclusion}

Using the rheological Johnson-Cook equation to depict the mechanical response of materials such as TA6V and HY100 reveals a softening effect of the stress attributed to self-heating of the material. From a thermodynamic standpoint, the existence of such softening leads to a strong thermomechanical coupling mechanism associated with a heat source superimposed on the viscoplastic dissipation. If the intrinsic dissipation is conventionally considered in the simulations using a viscoplastic energy rate computation weighted by a Taylor-Quinney coefficient, the heat source, induced by the material thermosensitivity, is to our knowledge most of the time not considered. Through some reasonable assumptions, it is possible to integrate Johnson-Cook's equation in a GSM model and then to calculate all terms of the energy balance associated with the development of ASBs. Superposition of the two heat effects first leads to temperature rises much closer to those observed experimentally. In addition, the energy analysis shows that, at very large strain, the overall heat source may become negative, resulting in a drop in temperature at the test end. This temperature decrease was also observed experimentally, which seems to confirm the role of the thermomechanical coupling source associated with thermal softening in the development of adiabatic shear bands.

\section{References}

Bai, Y.L., Dodd, B., 1992. Adiabatic Shear Localization - Occurrence, Theories and Applications. Pergamon Press.

Bever, M.B., Holt, D.L., Litchener, A.L. The stored energy of cold work, Progress in Materials Science vol. 17, 1973.

Chrysochoos, A., 1985. Bilan énergétique en élastoplasticité grandes déformations. J. Mech. Th. App. 4 (5), 589-614.

Chrysochoos, A., Belmahjoub, F., 1992. Thermographic analysis of thermo-mechanical couplings. Arch. Mech. 44, 55-68.
Chrysochoos, A., Wattrisse, B., Muracciole, J.M., El Kaim, Y.E., 2009. Fields of stored energy associated with localized necking of steel. J. Mech. Mater. Struct. 4 (2), 245-262.

Duffy, J., Chi, Y.C., 1992. On the measurement of local strain and temperature during the formation of adiabatic shear bands. Mater. Sci. Eng. A 157 (2), 195-210.

Germain, P., Son Nguyen, Q., Suquet, P., 1983. Continuum thermodynamics. J. Appl. Mech. Trans. ASME 50 (4b), 1010-1020.

Giovanola, J.H., 1988. Adiabatic shear banding under pure shear loading part ii: fractographic and metallographic observations. Mech. Mater. 7 (1), 73-87.

Halphen, B., Son Nguyen, Q., 1975. Sur les matériaux standard généralisés. J. Mécanique 14, 39-63.

Hartley, K.A., Duffy, J., Hawlew, R.H., 1987. Measurement of the temperature profile during shear band formation in steels deforming at high strain rates. J. Mech. Phys. Solids 35 (3), 283-301.

Johnson, G.R., Cook, W.H., 1983. A constitutive model and data for metals subjected to large strains, high strain rates and high temperatures. In: 7th International Symposium on Ballistics, The Hagues, Netherlands, pp. 541-547.

Johnson, G.R., Cook, W.H., 1985. Fracture characteristics of three metals subjected to various strains, strain rates, temperatures and pressures. Eng. Fract. Mech. 21 (1), 31-48.

Liao, S.C., Duffy, J., 1998. Adiabatic shear bands in a Ti-6Al-4V titanum alloy. J. Mech. Phys. Solids 46 (11), 2201-2231.

Longère, P., Dragon, A., 2009. Inelastic heat fraction evaluation for engineering problems involving dynamic plastic localization phenomena. J. Mech. Mater. Struct. 4 (2), 319-349.

Marchand, A., Duffy, J., 1988. An experimental study of the formation process of adiabatic shear bands in a structural steel. J. Mech. Phys. Solids 36 (3), 251-283.

Moreau, J.J., 1962. Fonctions convexes duales et points proximaux dans un espace hilbertien. C.R. Acad. Sci. 255, 2897-2899.

Moreau, J.J., 1963. Inf-convolution des fonctions numériques sur un espace vectoriel. C.R. Acad. Sci. 256, 1069-1071.

Oliferuk, W., Gadaj, S.P., Grabski, M.W., 1985. Energy storage during the tensile deformation of armco iron and austenitic steel. Mat. Sci. Eng. 70, 131-141.

Osvski, S., Rittel, D., Landau, P., Venkert, A., 2012. Microstructural effets on adiabatic shear band formation. Scripta Mater. 66, 9-12.

Osvski, S., Rittel, D., Venkert, A., 2013. The respective influence of microstructural and thermal softening on adiabatic shear localization. Mech. Mater. 56, 11-22.

Ranc, N., 2004. Etude des champs de température et de déformation dans les matériaux métalliques sollicités à grande vitesse de déformation, Ph.D., University of Paris 10

Ranc, N., Raynal, R., Taravella, L., Pina, V., Hervé, P., 2006. Theoretical and numerical study of strain localization under high strain rate solicitation. J. Phys. IV 134, 325-330. 
Ranc, N., Taravella, L., Pina, V., Herve, P., 2008. Temperature field measurement in titanium alloy during high strain rate loading-adiabatic shear bands phenomenon. Mech. Mater. 40 (4-5), 255-270.

Rittel, D., 1999. On the conversion of plastic work to heat during high strain rate deformation of glassy polymers. Mech. Mater. 31 (2), 131139.

Rittel, D., Wang, Z.G., 2008. Thermo-mechanical aspects of adiabatic shear failure of AM50 and Ti6Al4V alloys. Mech. Mater. 40 (8), 629635.

Rittel, D., Silva, M.L., Poon, B., Ravichandran, G., 2009. Thermomechanical behavior of single crystalline tantalum in the static and dynamic regime. Mech. Mater. 41 (12), 1323-1329.
Rittel, D., Kidane, A., Alkhader, M., Venkert, A., Landau, P., Ravichandran, G., 2012. On the dynamically stored energy of cold work in pure single crystal and polycrystalline copper. Acta Mater. 60 (9), 3719-3728.

Rosakis, P., Rosakis, A., Ravichandran, G., Hodowany, J., 2000. A thermodynamic internal variable model for the partition of work into heat and stored energy in metals. J. Mech. Phys. Solids 48 (3), 581-607.

Su, S., 2012. Energy-based variational modelling of adiabatic shear band structure, Ph.D., Ecole Centrale de Nantes.

Taylor, G.I., Quinney, H., 1934. The latent energy remaining in a metal after cold working. Proc. R. Soc. A143, 307-326.

Zener, C., Hollomon, J.H., 1944. Effect of strain rate upon plastic flow of steel. J. Appl. Phys. 15, 22-32. 